\title{
Archéopages
}

Archéopages

Archéologie et société

40 | 04-07/2014

Villages

\section{Les remplissages de deux puits d'un temple à Magalas}

Olivier Ginouvez

\section{(2) OpenEdition}

1 Journals

Édition électronique

URL : https://journals.openedition.org/archeopages/654

DOI : 10.4000/archeopages.654

ISSN : 2269-9872

\section{Éditeur}

INRAP - Institut national de recherches archéologiques préventives

\section{Édition imprimée}

Date de publication : 1 juin 2015

Pagination : 165-166

ISSN : $1622-8545$

\section{Référence électronique}

Olivier Ginouvez, «Les remplissages de deux puits d'un temple à Magalas », Archéopages [En ligne],

40 | 04-07/2014, mis en ligne le 01 juillet 2016, consulté le 24 janvier 2022. URL : http://

journals.openedition.org/archeopages/654 ; DOI : https://doi.org/10.4000/archeopages.654 
Le puits en milieu urbain

Odile Maufras, Inrap, UMR 5140

«Archéologie des sociétés méditerranéennes"

Le milieu urbain se caractérise par deux traits qui limitent le succès de l'enquête archéologique. L'un est la mauvaise conservation des vestiges anciens que la multitude des caves modernes a fait disparaître. Les élévations antiques et médiévales sont rarement conservées, les sols et une partie du sous-sol manquent. Ne restent de ces périodes que les aménagements qui impactent très profondément le terrain: fondations des constructions monumentales et puits. Autre trait: la densité et l'imbrication des vestiges sur plusieurs mètres d'épaisseur.

Dans ce contexte, les puits sont des structures prometteuses. Aménagés sur une grande profondeur, ils recèlent un volume de plusieurs mètres cubes de vestiges largement issus de la surface et susceptibles de documenter les parties disparues des sites. Ainsi, l'archéologue n'aura de cesse que de curer les puits. Cependant, leur fouille n'est pas aisée en milieu urbain. Le puits n'y est pas isolé, aussi ne peut-on envisager de le traiter manuellement par tranches de 1,30 $\mathrm{m}$ de profondeur avec élargissement mécanique de la fenêtre de fouille par autant de paliers de sécurité que de tranches, sans avoir au préalable fouillé l'intégralité des vestiges environnants. Et cela sur une surface d'autant plus vaste que le puits est profond. Or, en milieu urbain, les surfaces de fouille sont restreintes. En 1998, à Montpellier, un puits à roue de l'hôpital du Saint-Esprit a été mis au jour (Maufras, 1999). Sa fouille par paliers n'a pas été praticable, à la fois par la présence tout alentour d'un cimetière dense, également faute de place [ill. 1]. Ici, l'équipe archéologique s'est contentée des quelques fragments d'architecture retrouvés dans le mètre supérieur pour illustrer les élévations de l'établissement. En 2005, aux abords de la cathédrale de Nîmes, c'est

l'interdiction de fouiller sous le niveau inférieur des fondations du projet d'aménagement, outre son emprise réduite, qui a interdit la réalisation de paliers. La structure d'Archéopuits n'existait pas encore et seul le mètre supérieur a été fouillé. Il a livré une partie de la superstructure du puits et l'on attendait beaucoup de la fouille plus profonde qui n'a pu être réalisée (Maufras et al., 2007). En 2011, l'opération menée sur une partie du couvent des Carmes de Nîmes a mis au jour deux puits du $\mathrm{I}^{\mathrm{er}}$ siècle avant notre ère. Leur fouille avait été prévue, dès l'élaboration du projet d'intervention, et envisagée en fin de chantier. Ils n'ont été accessibles que lorsque leur environnement a été fouillé sur la surface nécessaire à la mise en place de la plate-forme, de la station de tamisage au stockage des terres extraites et à la circulation d'un engin, soit 30 à $40 \mathrm{~m}^{2}$ [ill. 2]. La fouille de ces puits a semblé décevante dans un premier temps : l'abaissement de la nappe phréatique n'a pas permis la conservation des matières organiques et aucun élément architectural n'y a été rejeté. Nous n'avons donc collecté que peu d'indices sur l'environnement paysagé et monumental du site, et aucun objet ou aménagement en bois, cuir ou tissu. Cependant, le dépôt abondant de vaisselle dans l'un des puits a permis de saisir la longue durée d'utilisation que les niveaux de surface ne permettaient pas de déceler tandis que l'autre, aménagé dans un espace d'atelier que l'on croyait voué à la tannerie, a livré quelques ossements qui ont mis en évidence une activité de pelleterie, notamment de fourrure d'hermine, que nous n'avions pas soupçonnée.

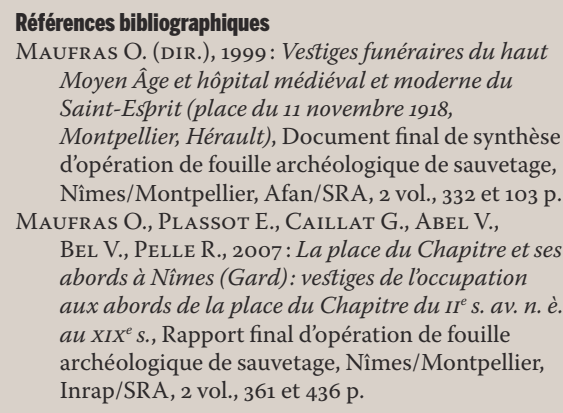

\section{Les remplissages de deux puits} d'un temple à Magalas

Olivier Ginouvez, Inrap, UMR 5140

«Archéologie des sociétés méditerranéennes »

Le site archéologique des terrasses de Montfau avoisine le pied d'un important oppidum fréquenté à partir du $\mathrm{VI}^{\mathrm{e}}$ siècle avant notre ère. Fouillée anciennement, l'agglomération protohistorique occupe les abords d'un itinéraire important reliant le littoral au rebord méridional du Massif central, via la cité de Béziers établie à $20 \mathrm{~km}$ plus au sud. Sur une surface de $9300 \mathrm{~m}^{2}$, l'étude a concerné les vestiges d'un sanctuaire avoisiné par une série de bâtiments vraisemblablement voués à l'accueil et à l'hébergement des pèlerins. Les premiers aménagements sont pour l'instant datés du début $\mathrm{du} \mathrm{I}^{\mathrm{er}}$ siècle avant notre ère et consistent en un vaste enclos quadrangulaire, délimité par un fossé comblé de fragments d'amphores italiques. Le site répond dès cette date à une fonction religieuse que les remaniements à venir ne feront que maintenir, au prix de modifications architecturales sans cesse renouvelées jusqu'au début du III $^{\mathrm{e}}$ siècle. Le sanctuaire primitif est matérialisé par son périmètre fossoyé. Un puits, quelques fosses isolées et deux fours à céramique lui sont également associés, mais le seul temple identifié dans le cadre de la fouille relève de la phase suivante. Peu après le changement d'ère, un mur maçonné est substitué au fossé précédent. Le nouveau péribole comprend dès lors un bâtiment de culte et 


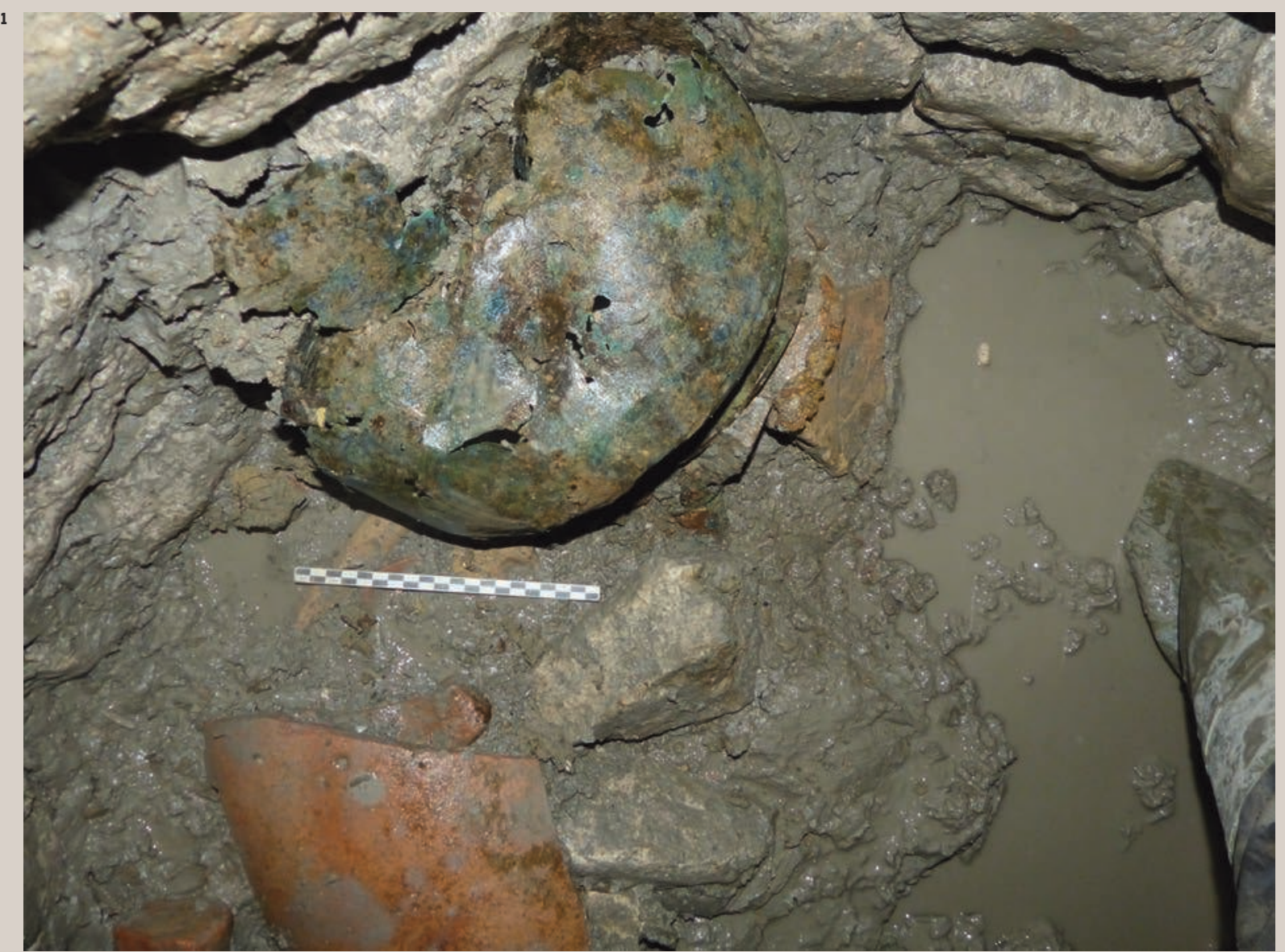

un long portique adossé à la limite septentrionale de la grande esplanade.

Deux des cinq puits mis au jour sur le site ont fait l'objet d'une étude intégrale, les moyens alloués à l'opération ne permettant pas d'envisager de fouiller l'ensemble des structures. Les constructions choisies l'ont été en raison de leur attribution chronologique: chacune correspondant à l'une des deux phases d'occupation déduite de l'étude stratigraphique. Il était par conséquent possible d'escompter la découverte d'indices en mesure d'argumenter non seulement le mode de fonctionnement du temple voisin (dédicaces, offrandes...), mais également les éventuelles spécificités du vaisselier réservé au service des pèlerins - en sus, bien sûr, des informations liées à leur alimentation et des éclairages paléoenvironnementaux attendus de la découverte de graines ou de restes de fruits. Le puits le plus ancien s'est avéré décevant. Profond de $7 \mathrm{~m}$ et dépourvu d'enveloppe construite, il n'a livré que très peu de renseignements. Seule la partie sommitale du remplissage incluait deux amphores italiques, ainsi que plusieurs centaines de tessons provenant de contenants vinaires du même type.

La seconde construction, en revanche, a révélé la présence d'une stratigraphie dont l'ensemble des recharges superposées sur une épaisseur de $17 \mathrm{~m}$ (couches de fonctionnement et remblais d'abandon intermédiaires) abritait un mobilier très abondant ainsi qu'une grande quantité d'objets en bois et de macro restes (fruits essentiellement: noix, noisettes, raisins...). Aucun élément en rapport direct avec le sanctuaire n'a été mis au jour. La céramique est foisonnante. Une quinzaine de récipients complets a été remontée en surface (dont trois en bronze) [ill. 1], ainsi que plusieurs milliers de tessons dont l'étude (à venir) permettra de faire la part entre les matériels de puisage (des cruches le plus souvent) et les vases de stockage qui permettaient d'immerger certains aliments, ainsi conservés quelque temps dans la fraîcheur du réservoir aménagé dans la partie inférieure du conduit.

\footnotetext{
Références bibliographiques

Ginouvez O., 2013: Hérault, Magalas, Le Pendut. Projet de lotissement Les terrasses de Montfau, Rapport final d'opération, diagnostic archéologique, Inrap Méditerranée.
}

\section{Chronologie de l'occupation de la villa de la Lesse à Sauvian basée sur l'étude des céramiques issues des puits \\ Pierre Rascalou, Inrap, UMR 5140 «Archéologie des sociétés méditerranéennes»}

La fouille de puits apporte généralement un volume considérable de mobiliers. Pour le céramologue, cela signifie la gestion de nombreuses caisses encombrantes et l'étude de milliers de tessons appartenant à quelques 